\title{
A New Technique for Solving Transportation Problems by Using Decomposition-Based Pricing and its Implementation in Real Life
}

\author{
Sajal Chakroborty and M. Babul Hasan* \\ Department of Mathematics, Dhaka University, Dhaka-1000, Bangladesh
}

(Received: 22 June 2015; Accepted: 24 December 2015)

\begin{abstract}
In this paper, we develop a new technique for solving transportation problems (TP) and develop a computer code by using mathematical programming language AMPL. There are many existing techniques for solving TP problems in use. By these techniques one has to determine initial basic feasible solution at first then improve this solution to determine optimal solution by another method. But this process is very lengthy and time consuming. By our technique we can determine optimal solution directly without determining initial basic feasible solution and optimal solution separately and we hope that this technique will provide an easier way than that of the other methods. We use the idea of decomposition based pricing (DBP) method to develop our technique. To our knowledge, there is no other paper which used DBP to solve TP. We demonstrate our technique by solving real life models developed by collecting data from a business organization of Bangladesh.
\end{abstract}

Key words: LP, TP, Decomposition, DBP, AMPL.

\section{Introduction}

Transpiration problem (TP) is a special type of linear programming (LP) problem. It deals with the situation in which a particular commodity is shipped from a source to a destination. The transportation cost on a given route is directly proportional to the number of units transported in that route. Idea of TP can be easily extended to apply in the area of inventory control, employment scheduling, personnel assignment, cash flow statements and many others ${ }^{1}$. It is one of the most important topics of operations research (OR).

TP was first formulated by the French mathematician Grapard Monge in 1781. But major advances in this field were made by Russian mathematician Leonid Kantorvich ${ }^{2}$ during World War II.

\section{Paper Outline}

In this paper, we first discussed about some basic definitions and ideas related to our work and exhibit some existing solution procedures of TP in Section II and III respectively. After that we present the algorithm in Section IV and AMPL code of our developed technique in Section V. Then we present our developed balanced TP model and its solution by our developed technique in Section VI. We also present a discussion about this solution in Section VII. Then extend the idea and developed an unbalanced TP model in Section VIII. We also solved this by using our technique and present a discussion about the solution in this Section. Finally, we draw a conclusion about our work.

\section{Preliminaries}

In this section, we discuss some necessary definitions and techniques relevant to our work.

\section{Formulation of TP as LP}

We can formulate TP as a LP problem ${ }^{3}$ by defining $x_{i j}$ as the quantity shipped from source $i$ to destination $j$. Since $i$ can assume values from $1,2, \ldots, m$ and $j$ from $1,2, \ldots, n$ the number of decision variables is given by the product of $m$ and n. A mathematical formulation of TP as LP has presented below ${ }^{4}$.

\footnotetext{
*Author for correspondence. e-mail: mbabulhasan@yahoo.com
}

$$
\begin{aligned}
& \text { Minimize } z=\sum_{i=1}^{m} \sum_{j=1}^{n} c_{i j} x_{i j} \\
& \text { subject to } \\
& \sum_{j=1}^{n} x_{i j} \leq a_{i}(i \text { for } i=1,2, \ldots . m) \\
& \sum_{i=1}^{m} x_{i j} \geq b_{j}(j \text { for } j=1,2, \ldots . \mathrm{n}) \\
& \left.x_{i j} \geq 0 \text { (Nonnegative restrictions for all pairs }(i, j)\right)
\end{aligned}
$$

The supply constraints guarantee that the total amount shipped from any source does not exceed its capacity ${ }^{5}$. The demand constraints guarantee that the total amount shipped to a destination meets the minimum demand at the destination. Excluding the non-negativity constraints ${ }^{6}$ the total number of constraints is $(m+n)$. When the total supply equals the total demand., i.e.,

$$
\sum_{i=1}^{m} a_{i}=\sum_{j=1}^{n} b_{j}
$$

Then every available supply at the sources will be shipped to meet the minimum demands at the destinations. Then all the demand constraints would become strict equations, and we have a standard transportation problem given by:

$$
\text { Minimize } z=\sum_{i=1}^{m} \sum_{j=1}^{n} c_{i j} x_{i j}
$$

subject to

$$
\begin{gathered}
\sum_{j=1}^{n} x_{i j}=a_{i} \\
\sum_{i=1}^{m} x_{i j}=b_{j} \\
x_{i j} \geq 0, i=1, \ldots, m, j=1, \ldots, n
\end{gathered}
$$


In the next section, we discuss classification of TP problems.

Classification of TP

There are two different types of TP problems. These are,

(i) Balanced transportation problem

(ii) Unbalanced transportation problem

\section{Balanced transportation problem}

When total supply is equal to the total demand, i.e,

$$
\sum_{i=1}^{m} a_{i}=\sum_{j=1}^{n} b_{j}
$$

Then the transportation problem is called balanced transportation problem. In the next section, we have discussed about unbalanced TP.

\section{Unbalanced transportation problem}

There are two types of unbalanced transportation problem ${ }^{7}$.

(i) When $\sum_{i=1}^{m} a_{i}>\sum_{j=1}^{n} b_{j} i . e$. the total supply exceeds the total demand. We can convert this problem to a standard problem by adding a dummy market or destination. In that case we assume that the demand of the dummy destination be $b_{n+1}=$ $\sum_{i=1}^{m} a_{i}-\sum_{j=1}^{n} b_{j}$ and its cost components $c_{i, n+1}=$ 0 for all $(i=1,2,3, \ldots \ldots m)$.

(ii) When $\sum_{j=1}^{n} b_{j}>\sum_{i=1}^{m} a_{i}$ i.e the total demand exceeds the total supply. We can convert this problem to a standard transportation problem by adding a dummy source. In that case the capacity of the dummy source is $a_{m+1}=\sum_{j=1}^{n} b_{j}-\sum_{i=1}^{m} a_{j}$ and $c_{m+1, j}=0$ i.e. cost components become zero for all $(j=1,2, \ldots \ldots n)$.

\section{Decomposition Based Pricing Method}

Decomposition based pricing (DBP) is a solution procedure of optimization problems and widely applicable for solving large scale optimization problems ${ }^{8}$. This procedure iteratively solves a relaxed sub-problem to identify potential entering basic columns ${ }^{9}$. The sub-problem is chosen to exploit special structure, rendering it is easy to solve ${ }^{10}$. Mamer and McBride ${ }^{11}$ developed DBP for multi-commodity flow problems. There are different types of decomposition techniques in use. Bender's decomposition, Dantzig-Wolfe decomposition, Decomposition based pricing, Triangular decomposition etc. are some decomposition techniques, ${ }^{8,12}$. But DBP is the latest and faster technique among these ${ }^{13,14}$. Consider the following LP problem ${ }^{10}$.

$$
\begin{aligned}
& \text { Maximize } z=C^{1} X_{1}+C^{2} X_{2}+\cdots+C^{n} X_{n} \\
& \text { subject to } \\
& \qquad \begin{array}{c}
A_{1} X_{1}+A_{2} X_{2}+\cdots+A_{n} X_{n} \leq b \\
B_{1} X_{1} \\
B_{2} X_{2} \quad b_{1} \\
\cdots \cdots \cdots
\end{array} \\
& B_{n} X_{n} \quad \leq b_{n} \\
& X_{1}, X_{2}, \cdots \cdots X_{n} \geq 0
\end{aligned}
$$

Steps of this algorithm are discussed in the following steps below ${ }^{14}$.

Step 1: Set iteration $\mathrm{k}=1$. We use three alternative methods to pick an initial set of prices $\lambda^{k}$.

(i) Start with $\lambda^{1}=0$. Or,

(ii) Start with $\lambda^{1}>0$ as the dual prices taken from the relaxed constraints of the LP relaxation. Or,

(iii) Start with $\lambda^{1}>0$ such that $\operatorname{Max}\left(C_{i}-\right.$ $\left.\lambda^{k} A_{i}\right)>0$.

Step 2: Solve the sub-problem,

$$
\begin{array}{cl}
S\left(x, \lambda^{k}\right): & \text { Maximize } C_{i} X_{i}-\lambda^{k}\left(A_{i} X_{i}-b\right) \\
& \text { subject to } \\
& B_{i} X_{i} \leq b_{i} \\
& X_{i} \geq 0
\end{array}
$$

for $X_{i}>0, i=1, \ldots n$ and put $\mathrm{i}$ in $I^{k}$.

Step 3: Solve the restricted master problem,

$$
\begin{aligned}
& \mathrm{M}(\mathrm{X}, \mathrm{k}): \quad \text { Maximize } z=\sum_{i=1}^{n} C_{i} X_{i} \\
& \text { subject to } \\
& \sum_{i=1}^{n} A_{i} X_{i} \leq b \\
& B_{i} X_{i} \leq b_{i} \\
& X_{i} \geq 0
\end{aligned}
$$

Step 4: For stopping criterion, we use two alternate methods.

(i) Stop when the objective value of the subproblem and the restricted master problem are equal i.e. $v\left(S^{k}\right)=v\left(M^{k+1}\right)$. Else set $\mathrm{k}=\mathrm{k}+1$, go to step-1.Or,

(ii) Stop when no new variables come into the restricted master. Else go to step-2.

\section{Chronicles of Solving TP}

In the current section, we discuss briefly about some existing techniques to determine solution of TP problems ${ }^{4}$.

\section{North-west corner rule}

Step 1: Select the upper left (North West) cell of the transportation table.

Step 2: If allocation made is equal to the supply available at the first source (in the first row) then move vertically down to the next cell.

If allocation made is equal to the demand of the first destination (in the first column) then move horizontally to the next cell ${ }^{15}$.

Step 3: Then continue this process until an allocation is made in the south east corner cell of the transportation table. 
The basic solutions obtained by this method may be far from optimal since the transportation costs are completely ignored.

\section{The least cost method}

Step 1: Select the cell having lowest unit cost in the entire table and allocate the minimum of supply or demand values in the cell.

Step 2: Then eliminate row or column in which supply or demand is exhausted. If both the supply and demand values are same, either of the row or column can be eliminated. In case the smallest unit cost is not unique, then select the cell where maximum allocation can be made.

Step 3: Repeat the process with next lowest unit cost and continue until the entire available supply at various sources and demand at various destinations is satisfied.

In general this method provides better solution as compared to the North-west corner rule ${ }^{16}$.

\section{Vogel's approximation method (VAM)}

Step 1: Calculate penalty for each row and column by taking the difference between the two smallest unit costs. This penalty or extra cost has to be paid if one fails to allocate the minimum unit cost.

Step 2: Select the row or column with the highest penalty or select the minimum unit cost of that row or column. Then allocate the minimum of supply or demand values of that cell. If there is a tie then select the cell where maximum allocation could be made.

Step 3: Adjust the supply or demand and eliminate the satisfied row and column. If a row and column are satisfied simultaneously then one of them is eliminated and the other one is assigned to zero value. Any row or column having zero supply or demand cannot be used in calculating for future penalties ${ }^{17}$.

Step 4: Repeat this process until all the supply sources and demand destinations are satisfied.

This method yields a better initial solution which sometimes may be the optimal solution ${ }^{4}$.

\section{Proposed Technique}

In this section, we present algorithm of our developed technique. We demonstrate our algorithm by the following steps.

Step 1: $n \leftarrow$ Set number of Sources.

Step 2: $m \leftarrow$ Set number of destinations.

Step 3: $c \leftarrow$ Set cost matrix for each sources and destinations.

Step 4: Formulate TP as LP.

Step 5: Relax complicating constraints.

Step 6: Apply idea of DBP.

Step 7: Check whether sub-problem value or master problem value become equal or not.
Step 8: If sub-problem value and master problem value become equal then stop. Otherwise repeat steps 6 to 7 .

\section{Computer Code}

In this section, we develop a computer code for our proposed technique. We have used a mathematical programming language AMPL ${ }^{18}$. Our code consists of AMPL model file, AMPL data file and AMPL run file. But only model file has been presented in this paper. If readers are interested then they may contact with the authors.

\section{AMPL model file}

param n; \# no of master sources

param m; \#no of destinations

param $c\{i$ in $1 . . n, j$ in $1 . . m\}$; \# cost matrix

param supply $\{\mathrm{i}$ in $1 . . n\}>=0$; \# amount of commodity can be supplied

param demand $\{\mathrm{j}$ in $1 . . \mathrm{m}\}>=0$; \# amount of commodity reqiured

check: $\operatorname{sum}\{\mathrm{i}$ in $1 . . \mathrm{n}\} \operatorname{supply}[\mathrm{i}]=\operatorname{sum}\{\mathrm{j}$ in $1 . . \mathrm{m}\}$ demand[j];

$\operatorname{var} x\{\mathrm{i}$ in $1 . . \mathrm{n}, \mathrm{j}$ in $1 . . \mathrm{m}\}>=0$;

param lemda $\{\mathrm{i}$ in $1 . . \mathrm{n}\}$ default 0 ;

\# Subproblem

\#-

minimize sub: $\operatorname{sum}\{\mathrm{i}$ in $1 . . \mathrm{n}, \mathrm{j}$ in $1 . . \mathrm{m}\} \mathrm{c}[\mathrm{i}, \mathrm{j}] * x[\mathrm{i}, \mathrm{j}]-\operatorname{sum}\{\mathrm{i}$ in 1..n $\}$ lemda[i]*(sum $\{\mathrm{j}$ in $1 . . \mathrm{m}\} \mathrm{x}[\mathrm{i}, \mathrm{j}]$-supply[i]);

subject to

$\operatorname{dest}\{\mathrm{j}$ in $1 . . \mathrm{m}\}: \operatorname{sum}\{\mathrm{i}$ in $1 . . \mathrm{n}\} \mathrm{x}[\mathrm{i}, \mathrm{j}]=\operatorname{demand}[\mathrm{j}]$;

\# Master Problem

\#-

param $\mathrm{cm}\{\mathrm{i}$ in $1 . . \mathrm{n}, \mathrm{j}$ in $1 . . \mathrm{m}\}$ default 0 ;

var $\mathrm{y}\{\mathrm{i}$ in $1 . . \mathrm{n}, \mathrm{j}$ in $1 . . \mathrm{m}\}>=0$ default 0 ;

minimize master: $\operatorname{sum}\{\mathrm{i}$ in $1 . . \mathrm{n}, \mathrm{j}$ in $1 . . \mathrm{m}\}$

$\mathrm{cm}[\mathrm{i}, \mathrm{j}]^{*} \mathrm{y}[\mathrm{i}, \mathrm{j}]$;

subject to

$\operatorname{ss}\{\mathrm{i}$ in $1 . . \mathrm{n}\}: \operatorname{sum}\{\mathrm{j}$ in $1 . . \mathrm{m}\} \mathrm{y}[\mathrm{i}, \mathrm{j}]=\operatorname{supply}[\mathrm{i}]$;

$\operatorname{dd}\{\mathrm{j}$ in $1 . . \mathrm{m}\}: \operatorname{sum}\{\mathrm{i}$ in $1 . . n\} y[i, j]=\operatorname{demand}[\mathrm{j}]$

\section{AMPL Output System}

Like other software's such as FORTRAN, MATHEMATICA, MATLAB, LINDO etc, AMPL has an intrinsic system to run code. In AMPL model file and data file have to write in different text files. Then one can generate a run file and have to call model and data file in that run file. AMPL has different solvers. In the Fig.1 and Fig.2, we have shown how to run AMPL graphically. 


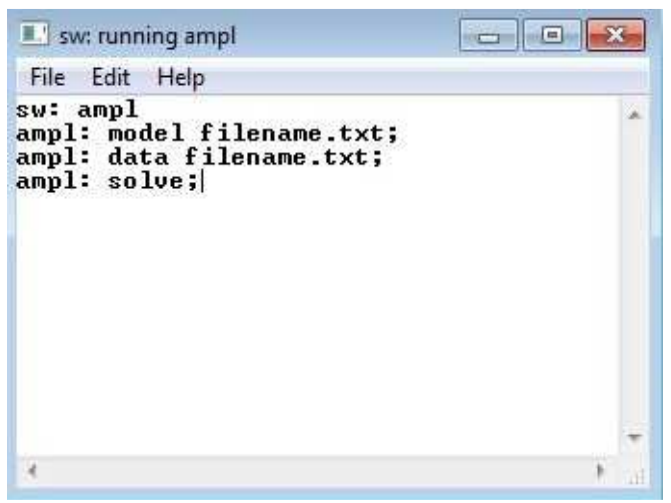

Fig.1. AMPL output system

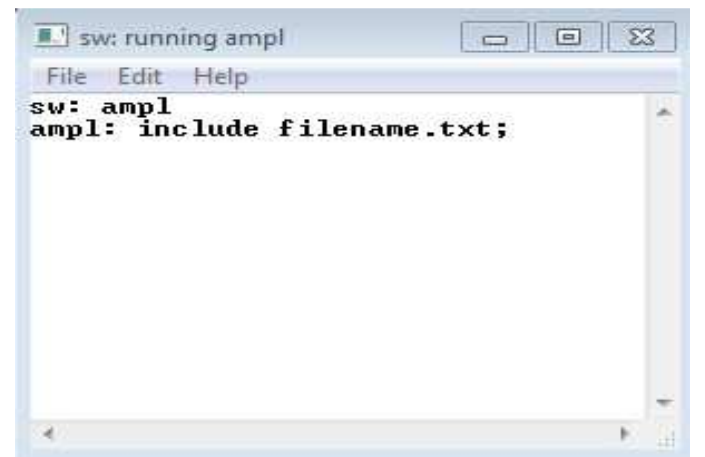

Fig.2. AMPL alternative running system

\section{Real Life Model}

In this section, we present a balanced TP problem. We develop this problem by collecting data from a business organization of Bangladesh.

\section{Balanced TP problem}

"Adil Publication" is one of the renowned book publishers of Bangladesh. They publish different types of text and other reference books related to science, literatures and history. Their books have lots of demand into markets. Buyers especially students like their published books due to easier and attractable representations of critical topics with various examples and figures of the relevant topics. "Adil Test Paper" is one of the most important reference book among these published by them. They have four different printing presses with book binders. They supply their books all over in Bangladesh. They want to minimize their transportation costs. In this model, we have considered only six different shops in different areas of Dhaka where they supply their reference book "Adil Test Paper". Required data has been presented in the following table.

Table 1. Data plan for balanced TP

\begin{tabular}{|c|c|c|c|c|c|c|c|}
\hline \multirow[b]{2}{*}{ From } & \multicolumn{6}{|c|}{ To } & \multirow{2}{*}{$\begin{array}{l}\text { Supply } \\
\text { (copies) }\end{array}$} \\
\hline & Shop 1 & Shop 2 & Shop 3 & Shop 4 & Shop 5 & Shop 6 & \\
\hline Press 1 & 21 & 16 & 25 & 13 & 22 & 31 & 11 \\
\hline Press 2 & 50 & 61 & 66 & 55 & 40 & 44 & 13 \\
\hline Press 3 & 80 & 85 & 91 & 87 & 99 & 91 & 19 \\
\hline Press 4 & 23 & 12 & 11 & 30 & 35 & 13 & 30 \\
\hline $\begin{array}{l}\text { Demand } \\
\text { (copies) }\end{array}$ & 6 & 10 & 12 & 15 & 10 & 20 & 73 \\
\hline
\end{tabular}

Total cost will be calculated in Taka. For example from Table 1, we see that required cost to shift books from Press 1 to Shop 1 is TK.21. To develop this model we have collected data from this company by direct communication.

\section{Formulation as LP}

To formulate the problem as a linear programming problem we begin by defining a variable for each decision that they must determine how much copies of book is sent from each press to each shop, we define

$x_{i j}=$ number of copies produced at press $i$ and sent to shop $j$ where $i=1,2,3,4$ and $j=1,2,3,4,5,6$.

$$
\begin{aligned}
& \text { Minimize } z=21 x_{11}+16 x_{12}+25 x_{13}+13 x_{14}+ \\
& 22 x_{15}+31 x_{16}+50 x_{21}+61 x_{22}+66 x_{23}+55 x_{24}+ \\
& 40 x_{25}+44 x_{26}+80 x_{31}+85 x_{32}+91 x_{33}+87 x_{34}+ \\
& 99 x_{35}+91 x_{36}+23 x_{41}+12 x_{42}+11 x_{43}+30 x_{44}+ \\
& 35 x_{45}+13 x_{46}
\end{aligned}
$$

\section{subject to}

$x_{11}+x_{12}+x_{13}+x_{14}+x_{15}+x_{16} \leq 11$

$x_{21}+x_{22}+x_{23}+x_{24}+x_{25}+x_{26} \leq 13$

$x_{31}+x_{32}+x_{33}+x_{34}+x_{35}+x_{36} \leq 19$

$x_{41}+x_{42}+x_{43}+x_{44}+x_{45}+x_{46} \leq 30$

Supply

Constraints

$x_{11}+x_{21}+x_{31}+x_{41} \geq 6$

$x_{12}+x_{22}+x_{32}+x_{42} \geq 10$

$x_{13}+x_{23}+x_{33}+x_{43} \geq 12$

$x_{14}+x_{24}+x_{34}+x_{44} \geq 15$

$x_{15}+x_{25}+x_{35}+x_{45} \geq 10$

$x_{16}+x_{26}+x_{36}+x_{46} \geq 20$

Demand

Constraints

$x_{i j} \geq 0, i=1,2,3,4$ and $j=1,2,3,4,5,6$

We observe that the problem is a balanced TP problem because sum of total supply is equal to the sum of total demand. 
We have considered supply constraints as complicating constraints. We can also consider demand constraints as complicating constraints. It's an alternative choice for the researcher. In this case we have to make a simple modification into the AMPL code. In the next section, we present optimal solution of the above problem by the AMPL code developed for our proposed technique.

\section{Optimal Solution by Our Proposed Technique}

We solve the real life problem of Section VI by our developed AMPL code. AMPL output has shown below.

\section{AMPL Output}

MINOS 5.5: optimal solution found.

4 iterations, objective 1053

MINOS 5.5: optimal solution found.

17 iterations, objective 1504

MINOS 5.5: optimal solution found.

4 iterations, objective 1504

MINOS 5.5: optimal solution found.

0 iterations, objective 1504

Variable values

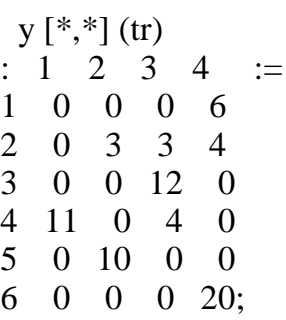

\section{Discussions}

From AMPL output, we have observed that minimum transportation cost is TK.1504 and from variable values we have observed the followings.

6 copies book have to transport from press 4 to shop 1 . 4 copies book have to transport from press 4 to shop 2 . 3 copies book have to transport from press 3 to shop 2 . 3 copies book have to transport from press 2 to shop 2 . 12 copies book have to transport from press 3 to shop 3 . 11 copies book have to transport from press 1 to shop 4 . 4 copies book have to transport from press 3 to shop 4 . 10 copies book have to transport from press 2 to shop 5 . 20 copies book have to transport from press 4 to shop 6 .

\section{Unbalanced TP Problem}

In this section, we have applied our method to test for solving an unbalanced TP. Let us consider the balanced TP problem described in Section VI. The problem was a balanced TP problem. Suppose the number of copies of books demanded is not 73. Suppose it is 61 . Then we see that total number of supplies is greater than total number of demands. Now according to the rule of TP we have to add a dummy shop. Then the Table 1 will be of the following form.

Table 2. Data plan for balanced TP

\begin{tabular}{|c|c|c|c|c|c|c|c|c|}
\hline \multirow[b]{2}{*}{ From } & \multicolumn{7}{|c|}{ To } & \multirow{2}{*}{$\begin{array}{l}\text { Supply } \\
\text { (copies) }\end{array}$} \\
\hline & Shop 1 & Shop 2 & Shop 3 & Shop 4 & Shop 5 & Shop 6 & Shop 7 & \\
\hline Press 1 & 21 & 16 & 25 & 13 & 22 & 31 & 0 & 11 \\
\hline Press 2 & 50 & 61 & 66 & 55 & 40 & 44 & 0 & 13 \\
\hline Press 3 & 80 & 85 & 91 & 87 & 99 & 91 & 0 & 19 \\
\hline Press 4 & 23 & 12 & 11 & 30 & 35 & 13 & 0 & 30 \\
\hline $\begin{array}{l}\text { Demand } \\
\text { (copies) }\end{array}$ & 6 & 10 & 12 & 15 & 10 & 8 & 12 & 73 \\
\hline
\end{tabular}

We solve this unbalanced TP by our own technique. Optimal solution of this problem has presented below.

\section{AMPL Output}

Optimal solution of the above unbalanced TP obtained by our method has given below.

MINOS 5.5: optimal solution found.

4 iterations, objective 897

MINOS 5.5: optimal solution found.

20 iterations, objective 1211

MINOS 5.5: optimal solution found.

4 iterations, objective 1211

MINOS 5.5: optimal solution found.

0 iterations, objective 1211

\section{Variable Values}

$\mathrm{y}[*, *](\operatorname{tr})$

$: \begin{array}{lllll}1 & 2 & 3 & 4 & :=\end{array}$

$\begin{array}{lllll}1 & 0 & 0 & 0 & 6\end{array}$

$\begin{array}{lllll}2 & 0 & 0 & 0 & 10\end{array}$

$\begin{array}{lllll}3 & 0 & 12 & 0 & 0\end{array}$

$\begin{array}{lllll}4 & 11 & 0 & 0 & 4\end{array}$

$\begin{array}{llllll}5 & 0 & 0 & 0 & 10\end{array}$

$\begin{array}{lllll}6 & 0 & 0 & 10 & 0\end{array}$

$\begin{array}{lllll}7 & 0 & 1 & 9 & 0\end{array}$ 


\section{Discussions}

From AMPL output, we have observed that minimum transportation cost is TK.1211 and from variable values we have observed the followings.

6 copies book have to transport from press 4 to shop 1 . 10 copies book have to transport from press 4 to shop 2 . 12 copies book have to transport from press 2 to shop 3 . 11 copies book have to transport from press 1 to shop 4 . 4 copies book have to transport from press 4 to shop 4 . 10 copies book have to transport from press 4 to shop 5 . 10 copies book have to transport from press 3 to shop 6 . 1 copy book has to transport from press 2 to dummy shop 7 . 9 copies book have to transport from press 3 to dummy shop 7 .

\section{Conclusion}

In this paper, we developed a new technique to solve TP problems. We used the idea of DBP to develop our technique. We also developed a real life oriented model by collecting data from a business organization of Bangladesh. First, we developed the model as a balanced TP problem. Then extended the idea and developed an unbalanced TP problem. We solved these models by using our technique. To our knowledge this is the first work about solution procedure of TP by using a decomposition technique specially DBP. We hope that this idea can be extended for some other works in future.

\section{References}

1. Winston, W. L., 1994. Linear Programming: Applications and Algorithm, Dunbury Press, Bellmont, California, U.S.A.

2. Kantorvich, L., 1942. On the Translocation of Masses, 37(2), 199-201.

3. Dantzig, G. B., 2002. Linear Programming, Operations research, 50(1), 42-47.

4. Ravindran, A., D. T. Phillips \& J. J. Solberg, Operations Research: Principles and Practice.
5. Haker, S., S. Angenent, and A. Tannenbaum, 2003. Minimizing Flows for the Mong, Kantorvich Problem. SIAM, 35(3), 61-97.

6. Gupta, P.K., D.S. Hira, 2005. Problems in Operations Research Principles and Solution, S.Chand \& Company LTD., New Delhi-110055, 406-484.

7. Taha, H. A., 2007. Operations research: An introduction, $8^{\text {th }}$ Ed. Pearson Princeton hall.

8. Dantzig, G. B., \& P. Wolfe, 1961. The Decomposition Algorithm for Linear Programming, Econometrica, 29(4), 767-778.

9. Hasan, M. B., \& J. F. Raffensperger, 2007. A Decomposition-Based Pricing Method for Solving A LargeScale MILP Model for an Integrated Fishery, Journal of Applied math and decision sciences, Article ID 56404.

10. Libbecke, M. E. \& J. Desrosiers, 2005. Selected topics in column generation, Operations research, 53(6), 1007-1023.

11. Mamer, J. W. \& R. D. McBride, 2000. A Decompositionbased Pricing Procedure for Large- Scale Linear Programs: An application to the linear multi-commodity Flow Problem, 46(5), 693-709.

12 Islam, A., M. B. Hasan, and H.K. Das, 2015. A New Decomposition-Based Pricing Technique for Solving LargeScale Mixed IP with A Computer Technique, 34(1), 5-20.

13. Conjeo, A. J., E. Castillo, R. Minguez, and R. G. Bertrand. Decomposition Techniques in Mathematical Programming.

14. Sweeny, D. J. \& R. A. Murphy, 1979. A method of Decomposition for integer programs, Operations research, 27(6), 1128-1141.

15. Frederich S. Hiller, Gerald J. Lieberman, 1995. Introduction of Operations research, Sixth edition, McGraw-Hill, New York, 350-404.

16. Gass, S. L., 2004. Linear Programming Method and Application, fifth edition, McFGraw-Hill Book Company, New York, 319-350.

17. Gupta, P. K., Man Mohan, 1997. Linear Programming and Theoty of Games, Eight editions, McGraw-Hill Book Company LTD, New Delhi-110002, 343-462.

18. www.ampls.com 
